\title{
Field studies of non-Bragg component variations of X-band radar return in the presence of surfactant films and intense long waves
}

\author{
I. A. Sergievskaya, S. A. Ermakov, A. V. Ermoshkin, I. A. Kapustin., O. V. Shomina \\ Institute of Applied Physics RAS, Nizhny Novgorod, 603950, Russia \\ E-mail: i.sergia@ipfran.ru
}

\begin{abstract}
Microwave dual-polarized radars are of great interest for remote sensing of the sea surface. According to modern point of view the microwave radar return at moderate incidence angles can be considered as a combination of a Bragg component due to scattering by resonant $\mathrm{cm}$-scale wind waves and a non-Bragg (nonpolarized) scattering component which is usually associated with wave breaking. At present, however, due to the lack of experimental data our understanding of the non-Bragg scattering is still insufficient to develop a quantitative model which could satisfactorily describe results of microwave probing of the sea surface. This paper presents new results of field observations of wind waves in the up- and cross-wind directions using a dual co-polarized X-band Doppler scatterometer at moderate incidence angles. It is found that non-Bragg scattering can be characterized by a "background" level and rare spikes, the period of which is several times larger than the period of dominant wind waves. Modification of the "background" level and the spikes in film slicks are analyzed.
\end{abstract}

Accepted: 15.09 .2020

DOI: 10.21046/2070-7401-2020-17-6-110-115

\section{Introduction}

The problem of backscattering of microwaves on the rough surface in application to radar remote sensing of the ocean upper layer has been actively discussed in the literature for some decades (see, for example, [1-7] and references therein). Some recent studies showed that traditional two-scale Bragg model cannot explain some important characteristics of radar backscatter, in particular, the relationship between the radar cross section values at vertical (VV) and horizontal (HH) co-polarizations. This leads to inability to characterize correctly various processes in the ocean upper layer such as marine currents, internal waves, film slicks etc. (see [10-18] and cited literature) using microwave imagery of the sea surface. It was hypothesized in [9] that some additional non-polarized or non-Bragg component strongly contributes in the radar return, especially at HH-polarization. The non-Bragg component can be associated with quasi specular reflection of microwaves from tilted facets which can appear due to features of different scales formed on the profile of nonlinear surface wind waves, including wave breaking features [8]. In [19] we have shown that the microwave radar backscatter at sufficiently high winds is determined essentially by strong wave breaking both at $\mathrm{HH}$ - and at VV-polarizations. It has been revealed that a ratio of the radar cross sections at VV- and $\mathrm{HH}$-polarizations (the polarization ratio) is unevenly distributed over the long wave profile both in slick and non-slick areas, and the polarization ratio values vary from 1 to the Fresnel coefficient ratio at corresponding polarizations. In this paper we analyse the variability of the contribution of strong wave breaking to non-Bragg component of radar return when observing the sea surface in cross- and upwind directions both for the cases of clean and film covered water surface. 


\section{Methods and instruments}

\subsection{Experiment}

The experiments were carried out from an Oceanographic Platform of the Marine Hydrophysical Institute (MHI) in May - June of 2019. A dual co-polarized (VV- and HH-polarizations) Doppler microwave radar and a three-band, $\mathrm{X} / \mathrm{C} / \mathrm{S}$-band Doppler radar were mounted at the Platform at heights of 12 and $13 \mathrm{~m}$, respectively, close to each other and looking nearly in the same directions. The antenna beam width of the X-band scatterometer is about $5.5^{\circ}$ and the observations were carried out at an incidence angle of $55^{\circ}$, so that the radar footprint scale was about $1.3 \mathrm{~m}$ for the X-band scatterometer, that is, about ten times less than the wavelengths of the dominant wind waves at wind velocities of $8-10 \mathrm{~m} / \mathrm{s}$. The X-band radar operated in a continuous regime. The sampling rate was $1000 \mathrm{~Hz}$ and Doppler spectra of radar return were analyzed over the time intervals of $0.25 \mathrm{~s}$. The radar Doppler shift was determined as a 'center of gravity' of the Doppler spectrum. The spatial and temporal resolutions for the scatterometer were essentially smaller than the wave lengths and periods of dominant wind waves (DWW) that allowed us to study variations of radar return over the DWW profile and all the results on the backscatter modulation were obtained with the X-band scatterometer. The X-channel of the three-band Doppler radar had the same antenna beam and was operating in a pulse regime. The temporal resolution of the three-band radar was comparable with the period of DWW, so the radar was used only to calibrate the $\mathrm{HH}$ and $\mathrm{VV}$ channels of the X-band scatterometer. The wind velocity and direction were measured with an acoustic anemometer WindSonic mounted at a height of 16 $\mathrm{m}$. Film slicks were created in the experiments using solutions of oleic acid in ethanol poured out on the water surface.

\subsection{Theoretical background}

The microwave radar return at moderate incidence angles is supposed to be determined by Bragg scattering due to resonant $\mathrm{cm}$-scale wind waves and by non-Bragg (unpolarized) scattering which is presumably associated with scattering from small quasi specular facets on the profile of surface waves longer than the Bragg ones. The quasi specular structures can appear due to nonlinearity of strong wind waves, in particular, due to wave breaking. The total normalized radar cross section (NRCS) $\sigma_{\mathrm{NBC}}$ can be written then as (see, for example, [8])

$$
\sigma_{p p}=\sigma_{p p}^{0}+\sigma_{N b} \text {. }
$$

Here $p$ denotes vertical $(\mathrm{V})$ or horizontal $(\mathrm{H})$ transmit/receive polarizations $\sigma_{p p}^{0}$ is Bragg (polarized) component of radar backscatter described by the Bragg model, $\sigma_{\mathrm{Nb}}$ is non Bragg component. The Bragg component according to the two-scale radar model can be written as (see, for example, [1])

$$
\sigma_{p p}^{0}=16 \pi k_{e m} g_{p p}^{2} F\left(k_{\mathrm{Br}}\right),
$$

where $F\left(k_{\mathrm{Br}}\right)$ is the wind wave spectrum at Bragg wavenumber $k_{\mathrm{Br}}=2 k_{e m} \cdot \sin \theta, k_{e m}$ is the wave number of an incident electromagnetic wave, $g_{p p}^{2}$ is the reflection coefficient which depends on polarizations of incident/reflected electromagnetic waves, $\theta$ is an incidence angle. Assuming that the non-Bragg component is non polarized, being the same for both $\mathrm{VV}$ and $\mathrm{HH}$ radar return, the non-Bragg component can be found from (1)-(2) as

$$
\sigma_{\mathrm{NBC}}=\frac{\sigma_{\mathrm{VV}}-R \sigma_{\mathrm{HH}}}{1-R},
$$

where $R=g_{\mathrm{VV}}^{2} / g_{\mathrm{HH}}^{2}$. The significant difference between $\mathrm{R}$ and polarization ratio $P R=\sigma_{\mathrm{VV}} / \sigma_{\mathrm{HH}}$ indicates a significant contribution of NBC in total NRCS.

The instantaneous radar Doppler shift can be found as a 'center of gravity' of the radar Doppler spectrum and can be written as $F_{D}=(1 / 2) k_{\mathrm{Br}}\left(V_{s}+V_{o r b}\right)$ (see, for example, [18]). Here $V_{s}$ denotes a velocity of scattering elements: either Bragg waves or scatterers associated with breaking wind waves and can also contain the velocity of large-scale and slowly varying surface currents, $V_{o r b}$ is the orbital velocity of DWW. 


\section{Results and discussion}

Figure 1a demonstrates that the polarization ratio weakly depends on the azimuth angle of observation, slightly decreasing towards the cross-wind observation. Figure $1 \mathrm{~b}$ illustrates the distribution of the polarization ratio along the profile of DWW. The ratio of mean radar returns at VV- and $\mathrm{HH}$-polarizations varies both for cross and upwind in the range from about 20-30 being roughly consistent with Bragg theory, to 1 that corresponds to purely non polarized scattering. Note that the Doppler shift changes in different limits for upwind and crosswind since the scatterers in upwind direction move with the orbital and the drift velocities, while the variation of Doppler shift in cross direction is mainly associated with a change in wave height. Figure 2 represents an example of time series of the intensity of NBC, which is characterized by a "background" level with strong and rare spikes. The five highest spikes account for $3 \%$ of the time and nearly $50 \%$ of the energy. The "background" is several times higher the noise level and takes $97 \%$ of the time and $50 \%$ of the energy. The modulation of the "background" is clearly visible. Figure 3 presents a record of the intensity of $\mathrm{NBC}$ for different conditions of observation. It shows that spikes in radar return are very intense, short and relatively rare. Simultaneous analysis of radar return and video of sea surface confirms that the spikes are caused by wave breaking visible by eyes, the frequency of spikes is several times less that the frequency of DWW. Note, that the spike intensities are close to each other in slick and in non slick areas both for upwind and crosswind observations, while the 'background' crosswind level is smaller than for an upwind direction. In slicks the 'background' level is less than on clean surface. Some conclusions regarding the role of spikes in the total radar backscatter can be made from histograms of radar backscatter maxima at $\mathrm{HH}$ - polarization presented in figure 4 and 5 . One can conclude that almost all maxima of the radar backscatter at $\mathrm{HH}$ polarization are contained within a narrow range of small intensity values (up to 5-10 arb. units in figure 5).
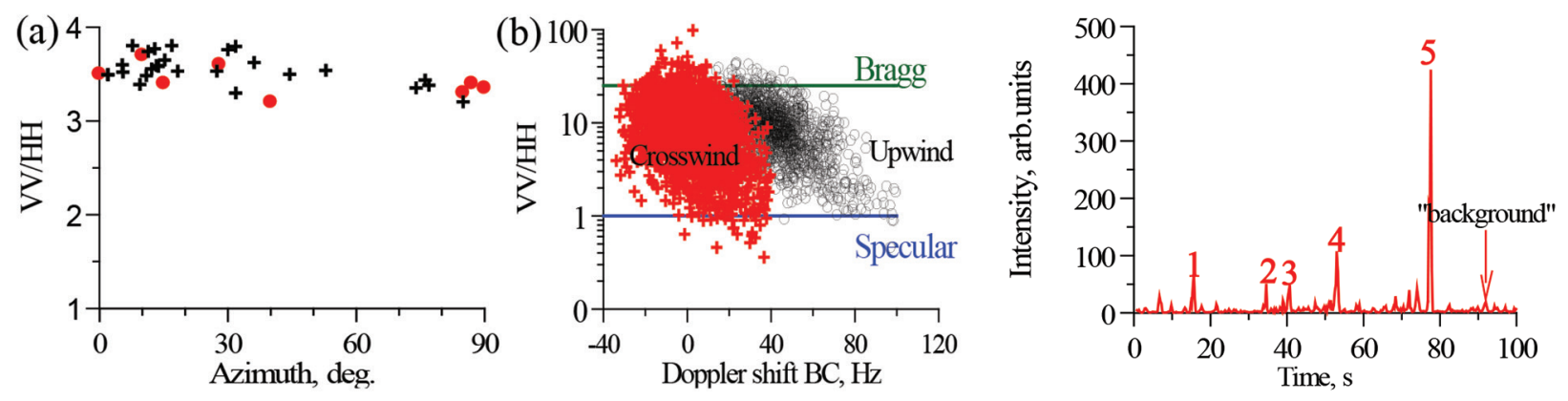

Figure 1. Polarization ratio vs. azimuth angle. Wind velocities $8-10 \mathrm{~m} / \mathrm{s}$. Incidence angle $55^{\circ}$. Black symbols $-\mathrm{X}$ channel of threeband radar, red $-\mathrm{X}$-band scatterometer (a); polarization ratio v.s. Doppler shift of a Bragg component for upwind and cross wind observation (b).

Figure 2. Time series of intensity of $\mathrm{NBC}$ of radar return. Upwind. $11 \mathrm{~m} / \mathrm{s}$.

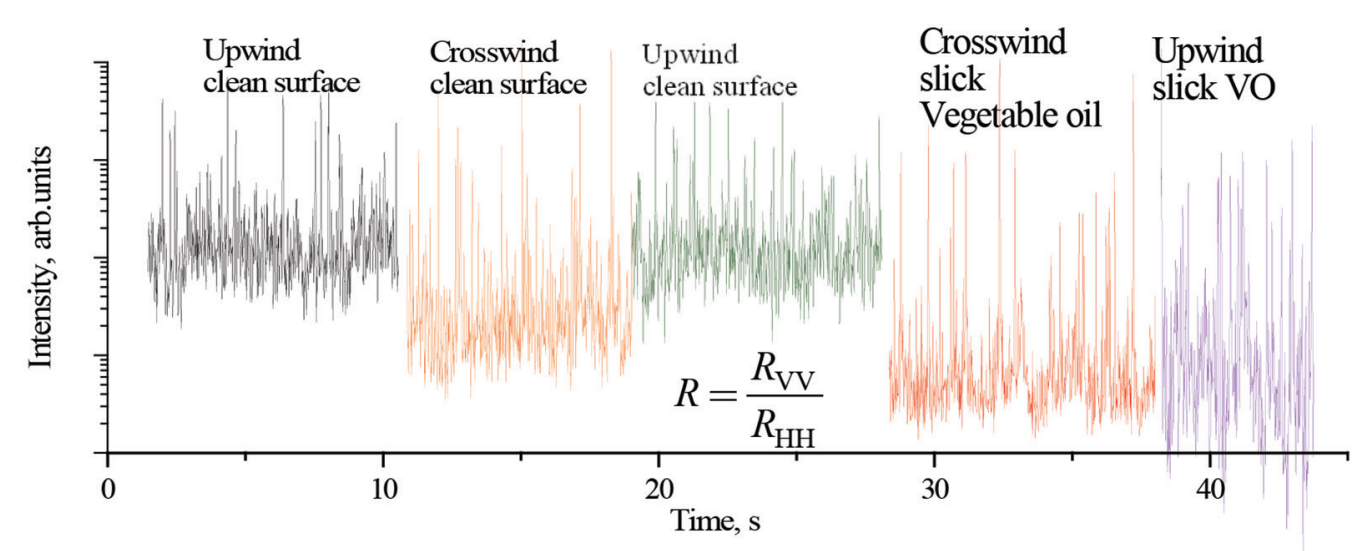

Figure 3. Intensity of NBC. 28.05.2019. $10 \mathrm{~m} / \mathrm{s}$. 

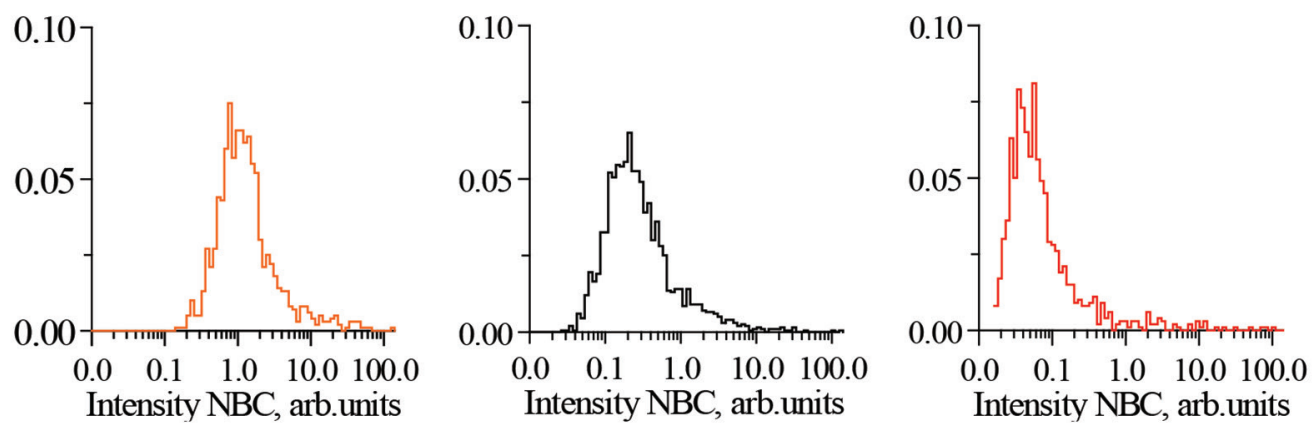

Figure 4. Histogram of backscatter maxima within a given intensity interval vs. intensity of local backscatter maximum: crosswind (left), upwind (middle), crosswind in slick (right).
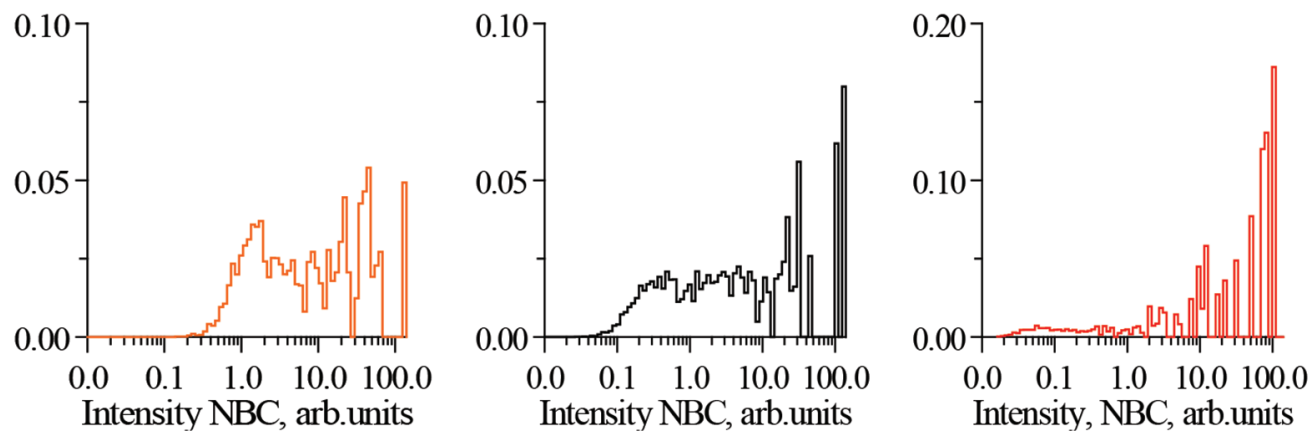

Figure 5. Histogram of total energy of local backscatter maxima within a given intensity interval vs. intensity of local backscatter maximum: crosswind (left), upwind (middle), crosswind in slick (right).

Figure 6a summarizes the results on variations of radar intensity from wave breaking areas and from the "background" at different azimuth angles. The data confirms that films do not significantly affect wave breaking, but strongly reduce the "background" level since the latter is determined by shorter waves. The obtained data give an additional argument supporting the hypothesis proposed in [8] that non-Bragg backscattering is isotropic. But in our case this can be applied only to strong wave breaking. Variation of the Bragg component (in the model this is variation of intensity of Bragg waves) strongly depends on azimuth angle. The latter is explained that at cross wind the Bragg waves are bound and their damping is determined by longer waves, which are weaker damped by surfactant films.
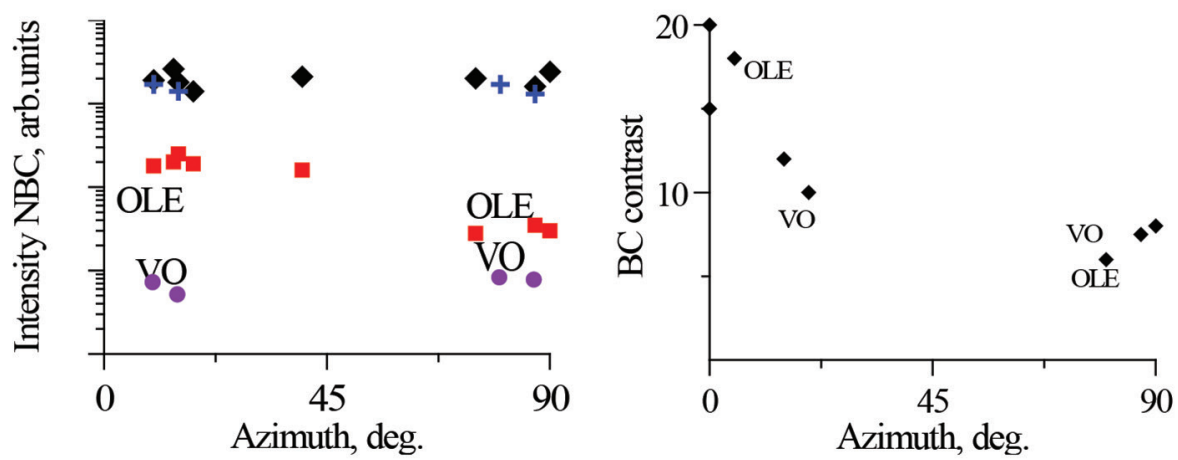

Figure 6. Radar return vs. azimuth angle. Black symbols - spikes, clean surface; blue - spikes, slick; red "background", clean surface; violet — "background", slick (a); Bragg radar component contrast (b).

Figure 7 illustrates variations of the Doppler shift of Bragg and non-Bragg components along the profile of DWW, here red symbols denote the Doppler shift in spikes which intensities are two times larger than the mean backscatter value for $\mathrm{HH}$-polarization. One can conclude that the most intense scatterers move with the highest velocities towards the radar both for crosswind and upwind cases. The similar situation is observed in slicks. 

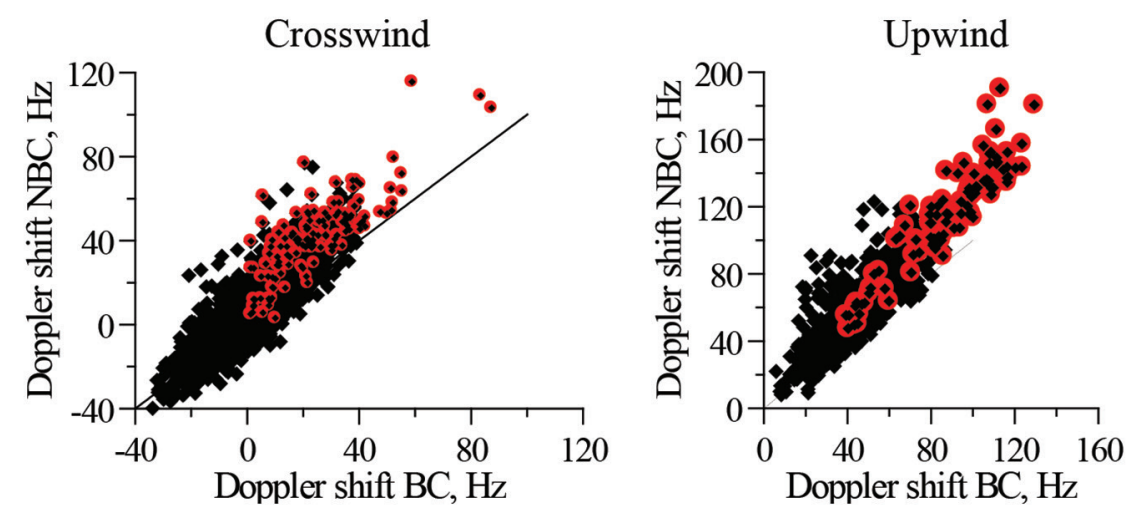

Figure 7. Doppler shift of BC\&NBC. Red symbols correspond to spikes.

\section{Conclusions}

1. At moderate to strong winds NBC is characterized by the "background" level and by strong and rare spikes which frequencies are less than the frequencies of DWW. The intensities of the spikes are more or less the same both for upwind and crosswind directions. The 'background level' for the upwind cases is several times larger than for the cross-wind direction. The ratio of the mean radar returns at $\mathrm{VV}$ - and $\mathrm{HH}$-polarizations varies along the profile of long wind wave in the range from about 20-30, being roughly consistent with Bragg theory, to 1 that corresponds to purely non polarized scattering. The spikes in radar return correspond to strong wave breaking ("whitecaps") moving toward the radar instrument.

2. In film slicks the spike intensity is comparable to the spikes for a clean water surface. The "background" level in slicks is lower than for clean surface and depends on the azimuth angle, the latter can be explained by the fact that the "background" level is determined mostly by shorter waves rather than the spikes.

\section{Acknowledgements}

This work was supported by the Russian Science Foundation (Project No. 18-17-00224).

\section{References}

[1] Valenzuela G., Theories for interaction of electromagnetic and oceanic waves: A review, Boundary-Layer Meteorology, 1978, Vol. 13, pp. 61-86.

[2] Bass F., Fuks M., Wave Scattering from Statistically Rough Surfaces, Oxford, UK: Pergamon, 1979.

[3] Donelan M., Pierson W., Radar scattering and equilibrium ranges in wind-generated waves with application to scatterometry, J. Geophysical Research, 1987, Vol. 92, pp. 4971-5029.

[4] Holliday D., St-Cyr G., Woods N. F., A radar ocean imaging model for small to moderate incidence angles, Intern. J. Remote Sensing, 1986, Vol. 7, pp. 1809-1834.

[5] Fung A., Li Z., Chen K., Backscattering from a randomly rough dielectric surface, IEEE Trans. Geosciences Remote Sensing, 1992, Vol. 30, pp. 356-369.

[6] Voronovich A. G., A two-scale model from the point of view of small-slope approximation, Waves Random Media, 1996, Vol. 6, pp. 73-83.

[7] Plant W., Microwave sea return at moderate to high incidence angles, Waves Random Media, 2003, Vol. 13, pp. 339-354.

[8] Kudryavtsev V., Hauser V., Caudal D., Caudal G., Chapron B., A semi-empirical model of the normalized radar cross-section of the sea surface. Part 1: The background model, J. Geophysical Research, 2003, Vol. 108(C3), 8054.

[9] Phillips O. M., Radar Returns from the Sea Surface - Bragg Scattering and Breaking Waves, J. Physics, 1988, Vol. 18, pp. 1065-1074.

[10] Jessup A., Keller W., Melville W., Measurements of sea spikes in microwave backscatter at moderate incidence, J. Geophysical Research, 1990, Vol. 95, pp. 9679-9688.

[11] Ermakov S., Kapustin I., Sergievskaya I., On peculiarities of scattering of microwave radar signals by breaking gravity-capillary waves, Radiophysics and Quantum Electronics, 2012, Vol. 55(7), pp. 453-461.

[12] Ermakov S., Kapustin I., Kudryavtsev V., Sergievskaya I., Shomina O., Chapron B., Yurovskiy Y., On the Doppler Frequency Shifts of Radar Signals Backscattered from the Sea Surface, Radiophysics and Quantum Electronics, 2014, Vol. 57, 239-250. 
[13] Reale F., Dentale F., Carratelli E., Numerical Simulation of Whitecaps and Foam Effects on Satellite Altimeter Response, Remote Sensing, 2014, Vol. 6, 3681-3692.

[14] Minchew B., Jones C. E., Holt B., Polarimetric analysis of backscatter from the Deepwater Horizon oil spill using L-band synthetic aperture radar, IEEE Trans. G. Remote Sensing, 2012, Vol. 50(10), pp. 3812-3830.

[15] Kudryavtsev V., Chapron B., Myasoedov A., Collard F., Johannessen J., On dual co-polarized SAR measurements of the Ocean surface, J. IEEE Geosciences Remote Sensing Letters, 2013, Vol. 10(4), pp. $761-765$.

[16] Hansen M., Kudryavtsev V., Chapron B., Brekke C., Johannessen J., Wave breaking in slicks: impacts on C-band quad-polarized SAR measurements, IEEE J. Selected Topics in Applied Earth Observations and Remote Sensing, 2016, Vol. 9(11), pp. 4929-4940.

[17] Ermakov S., Kapustin I., Lavrova O., Molkov A., Sergievskaya I., Shomina O., Damping of surface waves due to oil emulsions in application to ocean remote sensing, Proc. SPIE'2017, 2017.

[18] Ermakov S., Sergievskaya I., da Silva J., Kapustin I., Shomina O., Kupaev A., Molkov A., Remote Sensing of Organic Films on the Water Surface Using Dual Co-Polarized Ship-Based X-/C-/S-Band Radar and TerraSAR-X, Modulation of Dual-Polarized X-Band Radar Backscatter Due to Long Wind Waves, Remote Sensing, 2018, Vol. 10(7), 1097.

[19] Sergievskaya I., Ermakov S.A., Ermoshkin A.V., Kapustin I.A., Molkov A.A., Danilicheva O.A., Shomina O.V., Modulation of Dual-Polarized X-Band Radar Backscatter Due to Long Wind Waves, Remote Sensing, 2019, Vol. 11(4), 423. 